\title{
Small-Scale Drop Size Variability: Impact on Estimation of Cloud Optical Properties
}

\author{
Y. KnyaZikhin,* A. MarshaK, ${ }^{+}$M. L. Larsen,\#,@ W. J. Wiscombe, ${ }^{+}$J. V. MartonchiK, ${ }^{\&}$ \\ AND R. B. MYNENI* \\ *Department of Geography, Boston University, Boston, Massachusetts \\ + Climate and Radiation Branch, NASA Goddard Space Flight Center, Greenbelt, Maryland \\ \#Department of Physics, Michigan Technological University, Houghton, Michigan \\ @ Goddard Earth Science and Technology Center, University of Maryland, Baltimore County, Baltimore, Maryland \\ \&Jet Propulsion Laboratory, California Institute of Technology, Pasadena, California
}

(Manuscript received 9 February 2004, in final form 23 November 2004)

\begin{abstract}
Most cloud radiation models and conventional data processing techniques assume that the mean number of drops of a given radius is proportional to volume. The analysis of microphysical data on liquid water drop sizes shows that, for sufficiently small volumes, this proportionality breaks down; the number of cloud drops of a given radius is instead proportional to the volume raised to a drop size-dependent nonunit power. The coefficient of proportionality, a generalized drop concentration, is a function of the drop size. For abundant small drops the power is unity as assumed in the conventional approach. However, for rarer large drops, it falls increasingly below unity. This empirical fact leads to drop clustering, with the larger drops exhibiting a greater degree of clustering. The generalized drop concentration shows the mean number of drops per cluster, while the power characterizes the occurrence frequency of clusters. With a fixed total number of drops in a cloud, a decrease in frequency of clusters is accompanied by a corresponding increase in the generalized concentration. This initiates a competing process missed in the conventional models: an increase in the number of drops per cluster enhances the impact of rarer large drops on cloud radiation while a decrease in the frequency suppresses it. Because of the nonlinear relationship between the number of clustered drops and the volume, these two opposite tendencies do not necessarily compensate each other. The data analysis suggests that clustered drops likely have a stronger radiative impact compared to their unclustered counterpart; ignoring it results in underestimation of the contribution from large drops to cloud horizontal optical path.
\end{abstract}

\section{Introduction}

Drop size and drop spatial distribution determine photon-cloud interaction. The classical approach assumes that the number of drops of a given radius is proportional to volume with a drop size-dependent coefficient of proportionality, the drop concentration, which is a volume-independent function of the spatial point. This assumption underlies the derivation of the drop size density distribution function, from which one can derive the extinction coefficient and scattering phase function, which are in turn input to the radiative transfer equation.

Corresponding author address: Yuri Knyazikhin, Department of Geography, Boston University, 675 Commonwealth Avenue, Boston, MA 02215.

E-mail: jknjazi@crsa.bu.edu
In general, drop size distributions depend on the spatial scale over which they are sampled. Liu et al. (2002) pointed out that there is a "saturation scale" beyond which observed size distributions do not change much with further increases in averaging scale. At this and larger scales, distributions are statistically stationary, in the sense that stable sampling has been achieved. However, at the saturation scale drop distributions maximize the spectral entropy (Liu et al. 2002) and thus convey minimum information about drop spatial variability (Ash 1965). The use of such distributions in the radiative transfer equation, therefore, makes the equation insensitive to small-scale cloud variability. Another extreme is the case when drops are sampled at an infinitesimal scale. The drop distribution is then given by Dirac delta functions, which account for the total number of drops with specific radius and correspond to the minimum spectral entropy (Liu et al. 2002). Although 
information about drops conveyed by these distributions is maximized, the drop concentration cannot be defined at this scale and thus cannot be used in the radiative transfer equation. A Monte Carlo technique seems to be the only way to account for the full amount of information conveyed by such distributions.

Drop size distributions are strongly scale dependent when the sampling scale is between the infinitesimal and saturation scales. This suggests that drop distributions at these scales convey a considerable amount of information on drop variability. The radiative transfer equation, in turn, aims to relate small-scale properties of the medium to the photon distribution in the entire medium. The question then arises, how essential is this information? We will focus on the drop variability at the subsaturation scale. Specifically, we would like to know the following: 1) How can the small-scale drop variability be parameterized? 2) How can information on small-scale drop variability be incorporated into the radiative transfer equation? 3) What is the effect of ignoring small-scale variability on cloud optical properties? The objective of this paper is to address these questions.

This paper is organized as follows. In sections 2 and 3 , aircraft data on liquid water drop sizes measured by the Forward Scattering Spectrometer Probe (FSSP) during the First International Satellite Cloud Climatology Project (ISCCP) Regional Experiment (FIRE), July 1987, and the Atmospheric Radiation Measurement (ARM) Program Cloud Intensive Operational Period (IOP), March 2000 (Dong et al. 2002), are analyzed to answer the first question. We propose a new approach of data processing in which statistical parameters are accumulated only over samples containing drops. By counting only the FSSP bins filled with drops, one can extract two pieces of information needed to quantify the drop variability: the occurrence frequency of samples of a given volume containing drops of a given size (section 2) and drop size-dependent mean number of drops in such samples (section 3). Both pieces, in turn, can be compressed into power-law relationships between the mean number of drops and volume (section 3). The conventional data processing techniques are based on the assumption that the mean number of drops is proportional to volume; thus the exponent in power-law relationships is unity. This assumption holds true for saturation or coarser sampling volumes. The analysis of microphysical data shows that, for smaller sampling volumes, the number of cloud drops of a given radius is instead proportional to a drop size-dependent nonunit power of the volume. In a companion paper (Marshak et al. 2005), we have shown that drop clustering is primarily responsible for devia- tion of the power from unity. An equation that describes the attenuation of the radiance and naturally accounts for the power-law statistics is derived in section 4. Nonlinear terms in this equation make traditional methods of solution inapplicable; however we were able to find an analytic solution by using new methods for "fractional" differential equations (appendixes $\mathrm{A}$ and $\mathrm{B}$ ). This solution is used to examine the effect of small-scale drop variability on cloud optical properties. Section 5 summarizes the results.

\section{Frequency of drop occurrence}

Two 10-min samples of the cloud drop size distribution measured by the FSSP during FIRE (Albrecht et al. 1988) are used in our analysis. The measurements were taken on 10 July 1987 over the Pacific Ocean off San Diego, California (King et al. 1990). Two flight legs are used; each of them is about $50 \mathrm{~km}$ long at an altitude of $725 \mathrm{~m}$ in the middle of a 440-m-thick marine stratocumulus cloud layer. The aircraft speed was 80 $\mathrm{m} \mathrm{s}^{-1}$. Drops were accumulated over 1-s time periods in order to form drop distributions consisting of 15 radius bins. The bin width is $2 \mu \mathrm{m}$; radii of the smallest and largest registered drop were 1.4 and $31.4 \mu \mathrm{m}$, respectively. In total, there were 1196 drop distributions; Fig. 1 shows an example of 100 samples. With the FSSP sample area $\varepsilon_{\min }^{2}=0.004 \mathrm{~cm}^{2}$ (Liu and Hallett 1998) and accumulation distance $l_{\min }=80 \mathrm{~m}$, each measurement provides the distribution of drop sizes in a volume $v_{\min }=l_{\min } \times \varepsilon_{\min }^{2}=32 \mathrm{~cm}^{3}$. We term the volume $v_{\min }$ the smallest FSSP collectable volume scale. The total volume, $V_{L}$, in which drops are sampled is $V_{L}=L \times$ $\varepsilon_{\min }^{2}$ where $L$ is the total length of the flight legs. Here and in section 3 , we focus on examining the distribution of drop sizes in the volume $V_{L}$, which is idealized as a "tube" with the base $\varepsilon_{\min }^{2}$ and the length $L$. For the FIRE FSSP data, $L=80 \mathrm{~m} \mathrm{~s}^{-1} \times 1196 \mathrm{~s}=95680 \mathrm{~m}$, and thus $V_{L}=38272 \mathrm{~cm}^{3}$.

Let $N(v, r)$ be the total number of drop distribution samples at a collectable volume scale $v=l \times \varepsilon^{2}$ containing drops with radii between $r-\Delta / 2$ and $r+\Delta / 2$ where $\Delta=2 \mu \mathrm{m}$ is the FSSP bin width. For the smallest FSSP collectable volume $v_{\min }$ the number $N\left(v_{\min }, r\right)$ can be evaluated from Fig. 1 by counting "nonempty" records in corresponding column. Each such record can contain more than one drop, but still adds only unity to $N$. This variable describes the frequency of the occurrence of drop with radius $r$ at a collectable volume scale $v$ along the flight legs. Here and in the rest of the text, $r$ refers to centers of the FSSP radius bins and represents drops with radii between $r-\Delta / 2$ and $r+\Delta / 2$. Our aim here is to examine variation in $N(v, r)$ as a function of the scale $v$. 

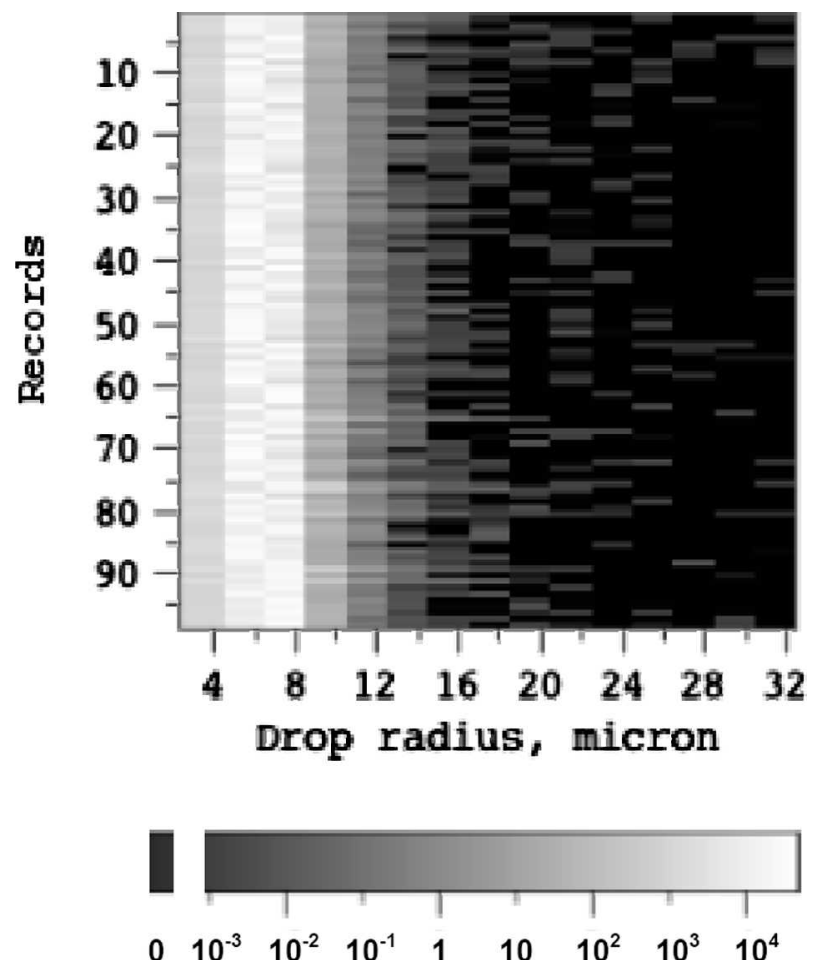

FIG. 1. One hundred drop size distribution samples measured by the FSSP on $10 \mathrm{Jul} 1987$ (King et al. 1990). The measurements were taken from 0844 to 0854 and 0931 to 0941 Pacific daylight time (PDT) over the Pacific Ocean off San Diego. The aircraft speed was $80 \mathrm{~m} \mathrm{~s}^{-1}$ and drops were accumulated over 1-s time period. Drops above $31.4 \mu \mathrm{m}$ are not registered. Vertical and horizontal axis show record number and centers of the FSSP radius bins, respectively. Each row corresponds to one sample of the drop size distribution. Columns show counts of drops of a given radius in 80-m intervals along the flight leg. Note that at a linear scale of $80 \mathrm{~m}, 14-\mu \mathrm{m}$ and larger drops do not appear in each sample.

At a scale comparable with the drop size, that is, when $v \rightarrow 0$, a bin has only one drop, so $\lim _{v \rightarrow 0} N(v, r)$ $=N(0, r)=N_{\text {Tот }}(r)$ will be the total number of drops registered in the bin $r$. Being defined as the number of samples of volume $v$ with nonempty bins, as $v \rightarrow 0$, a growing number of samples along the flight leg contribute nothing to the sample count $N(v, r)$. As a function of increasing scale $v, N(v, r)$ is nonincreasing. Indeed, an increase in $v$ lowers the number $N(v, r)$ of nonempty bins since nonempty bins can now contain more than one drop. In our case, $N(v, r)$ varies with $v$ between 1 ( $v$ $\left.=V_{L}\right)$ and the total number $N_{\mathrm{TOT}}(r)$ of drops with radius $r$ acquired in the volume $V_{L}$.

We define the average number of drops in the nonempty bin $r$ as

$$
\bar{n}(v, r)=\frac{\sum n(v, r)}{N(v, r)}=\frac{N_{\mathrm{TOT}}(r)}{N(v, r)} .
$$

The summation is performed over nonempty bins. Here $n(v, r)>0$ is the number of drops with radius $r$ in corresponding nonempty bin at a volume scale $v$. Their sum coincides with $N_{\text {TOT }}(r)$, which depends on the total volume, $V_{L}$, in which drops are collected rather than on the scale $v$ over which they are sampled. Thus,

$$
N_{\text {TOT }}(r)=N(v, r) \bar{n}(v, r) \text {. }
$$

This equation establishes an obvious but very important property of drop distribution: with a fixed total number, $N_{\text {TOT }}(r)$, of drops with radius $r$ in $V_{L}$, a decrease in the occurrence frequency, $N(v, r)$, at a given scale $v$ is necessary accompanied by an increase in the mean number, $\bar{n}(v, r)$, of drops per nonempty sample of the volume $v$. Since $N_{\text {Tот }}(r)$ does not depend on $v$, the product of two scale-dependent variables $\bar{n}(v, r)$ and $N(v, r)$ does not depend on $v$, either. Obviously, the total number of drops, $N_{\text {TOT }}(r)$, is a function of the volume $V_{L}$ in which drops are sampled. Since we focus on examining the distribution of drop sizes in the volume $V_{L}$, it is fixed throughout the paper. Variation in $N_{\text {TOT }}(r)$ with respect to $V_{L}$ is discussed in the companion paper (Marshak et al. 2005).

Next we degrade our FSSP data to longer accumulation distances of $l=2 l_{\min }, 3 l_{\min }, \ldots, 600 l_{\min }$ by joining two, three, etc. consecutive readings and estimate the frequency $N(v, r)$ at corresponding sampling volumes of $v=l \times \varepsilon_{\min }^{2}$. Figure 2 shows variation in $N(v, r)$ with respect to $v$. Two features can be clearly seen. First, beyond a volume scale of $v_{\text {sat }}=960 \mathrm{~cm}^{3}\left(l_{\mathrm{sat}}=2400 \mathrm{~m}\right)$, the number $N(v, r)$ of nonempty bins is inversely proportional to the volume $v$. At these scales, differences in drop variability cannot be discerned. Thus, in this case an accumulation distance of $2400 \mathrm{~m}$ is Liu et al.'s (2002) saturation scale. Once the saturation volume is exceeded, all drop bins will be occupied, and consequently the number of samples with nonempty bins becomes inversely proportional to the sampling volume. Second, for scales between $32 \mathrm{~cm}^{3}$ and about $960 \mathrm{~cm}^{3}$, variation in $N(v, r)$ also follows a power law, but with an exponent that is a function of drop size $r$, that is,

$$
N(v, r)=m\left(V_{L}, D\right) v^{-D(r)} .
$$

Here $m\left(V_{L}, D\right)=N(1, r)$ is the prefactor. Figure 2 provides a geometrical interpretation of $m\left(V_{L}, D\right)$; that is, the interception of the vertical axis of $\ln N$ by a straight line as a function of $\ln v, v<960 \mathrm{~cm}^{3}$, is given by $\ln \left[m\left(V_{L}, D\right)\right]$. The interception depends on the exponent $D(r)$ and the total volume $V_{L}$ where drops are collected, rather than on the scale $v$ over which they are sampled.

Figure 3a shows $D(r)$ as a function of drop size $r$. At scales between 32 and $960 \mathrm{~cm}^{3}$, small drops with 


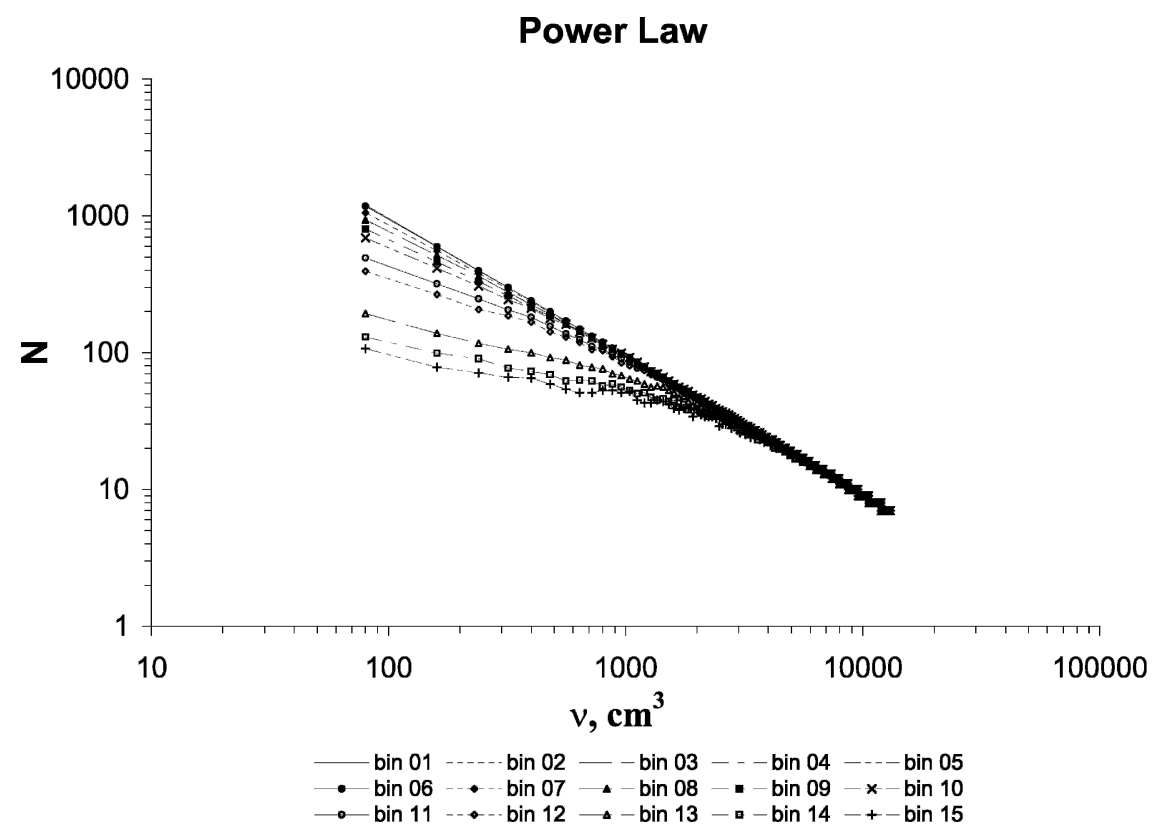

FIG. 2. Scaling behavior of bin count $N$ derived from data shown in Fig. 1. Legends indicate the FSSP radius bin numbers. At volume scales between 32 and $920 \mathrm{~cm}^{3}$, variation in $N(v, r)$ follows a power law with a drop size-dependent exponent $D(r)$, i.e., $N(v, r)=m\left(V_{L}, D\right) v^{-D(r)}$. Here $v=l \times \varepsilon_{\min }^{2}$ where $\varepsilon_{\min }^{2}=0.004 \mathrm{~cm}^{2}$ is the FSSP sampling area. The interception of the vertical axis of $\ln N$ by a straight line as a function of $\ln v$ is given by $\ln \left[m\left(V_{L}, D\right)\right]$ where $m\left(V_{L}, D\right)=N(1, r)$ is the prefactor. It depends on the exponent $D(r)$ and the total volume, $V_{L}=L \varepsilon_{\min }^{2}$, where drops are collected but not on the scale $v$ over which they are sampled. Here $L$ is the total length of the flight legs.

$r<14 \mu \mathrm{m}$ follow a power law with exponent $D(r)=1$. However, $D(r)$ for larger drops falls below unity. Figure 3 b complements Fig. 3a with values of $D(r)$ for drops larger than $30 \mu \mathrm{m}$. Note that this curve was derived from data collected at a different time (March 2000 versus July 1987), a different site (Oklahoma versus California), and using a different instrument (1D-C versus FSSP) and sampling strategy. Both figures illustrate an important behavior of $D(r)$ : for a given scale range, it is a nonincreasing function with respect to the drop (a)

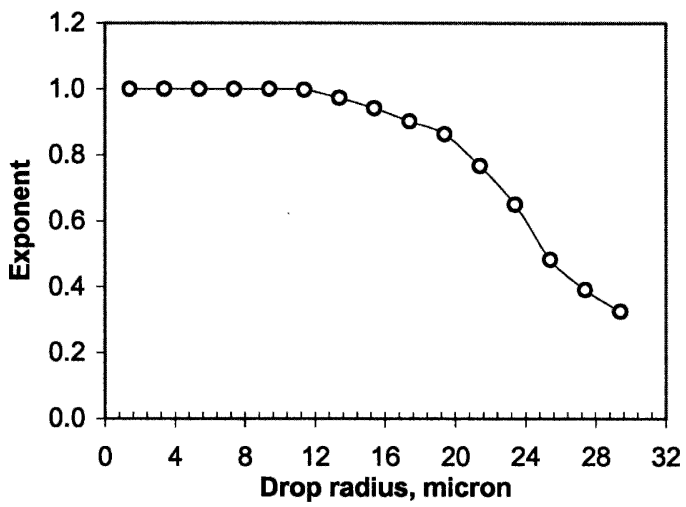

(b)

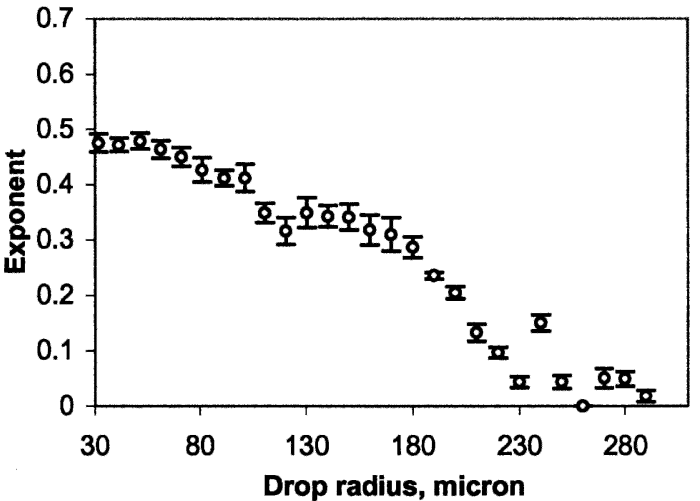

FIG. 3. (a) Exponent $D(r)$ as a function of drop radius in the range from 2 to $30 \mu \mathrm{m}$ derived from data acquired during the flight on $10 \mathrm{Jul} 1987$ as part of FIRE field program. (b) Exponent as a function of drop radius in the range from 30 to $300 \mu \mathrm{m}$. This curve was derived from data collected by the $1 \mathrm{D}-\mathrm{C}$ probe on board the University of North Dakota Citation aircraft during the ARM Cloud Intensive Operational Period (3 Mar 2000). These data are publicly available online at http://iop.archive.arm.gov/arm-iop/2000/sgp/cloud/poellot-citation/. 


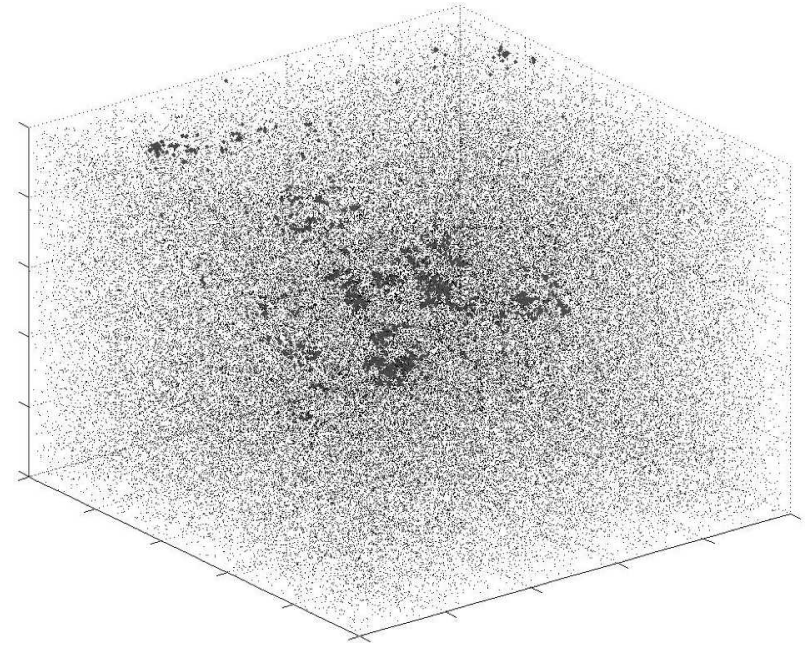

FIG. 4. Two examples of spatial distributions of 100000 small ( $D$ $=1$; colored gray $)$ and 1000 large $(D \approx 0.35$; colored black $)$ cloud drops. The numbers of small and large drops are in approximately the same proportion as corresponding numbers $N_{\mathrm{TOT}}(8)$ and $N_{\text {TOT }}(25)$ in the FSSP FIRE data. The small drops are distributed perfectly randomly $(D=1)$ whereas the large drops follow a power law with an exponent $D=0.35$ (Fig. 2). The deviation of the exponent $D$ from unity indicates drop clustering-the smaller its value is, the stronger clustering is.

size. The companion paper (Marshak et al. 2005) shows that this is due to the clustering of drops: the larger the droplet size, the greater the clustering. Examples of spatial distributions of 100000 small $(D=1)$ and 1000 large $(D \approx 0.35)$ cloud drops generated by a fractal model proposed by Marshak et al. (2005) are shown in Fig. 4. In these examples, $V_{L}$ is a cube of $33 \times 33 \times 33$ $\mathrm{cm}^{3}$. This figure clearly illustrates clusters of large drops. By a cluster here we understand any volume $v$ in $V_{L}$ that contains drops. Thus at a given scale $v, N(v, r)$ counts the numbers of clusters in $V_{L}$.

To summarize, it was found that 1 ) the occurrence frequency of drops follows a power law of the volume with a drop size-dependent exponent; 2) the exponent is a nonincreasing function with respect to the drop size and varies between 0 and $1 ; 3$ ) a deviation of the exponent from unity indicates drop clustering, with the larger drops exhibiting a greater degree of clustering; 4) the exponent characterizes the occurrence frequency of clusters-the lower its value, the rarer the occurrence.

\section{Generalized drop concentration}

We return to Eq. (1). Substituting (3) into (1), one obtains

$$
\bar{n}(v, r)=\frac{N_{\mathrm{TOT}}(r)}{m\left(V_{L}, D\right)} v^{D(r)}
$$

Note that for $v_{\min } \leq v \leq v_{\text {sat }}$ neither $N_{\text {TOT }}(r)$ nor $m\left(V_{L}\right.$, $D)$ vary with the scale $v$; as a result, their ratio is also scale independent. We will term the latter a generalized drop concentration, that is,

$$
\rho(r)=\frac{N_{\mathrm{TOT}}(r)}{m\left(V_{L}, D\right)} .
$$

Equation (4) can be rewritten as

$$
\bar{n}(v, r)=\rho(r) v^{D(r)} .
$$

In our example for volumes $v$ between $v_{\min }=32 \mathrm{~cm}^{3}$ and $v_{\mathrm{sat}}=920 \mathrm{~cm}^{3}$, the mean number of drops with radius $r$ in a nonempty volume $v$ is proportional to the volume raised to a drop size-dependent power.

Two variables are required to estimate the generalized drop concentration: the total number, $N_{\text {TOT }}(r)$, of drops with radius $r$ in the volume $V_{L}$ and the prefactor $m\left(V_{L}, D\right)$. The scale-independent prefactor can be specified not only from the interception (Fig. 2) but also from Eq. (3) as a product of two scale-dependent variables, namely,

$$
m\left(V_{L}, D\right)=N(v, r) v^{D(r)}, v_{\min } \leq v<v_{\text {sat }} .
$$

Figure 5 shows $m\left(V_{L}, D\right)$ as a function of $r$. For small drops $(r<14 \mu \mathrm{m}, D=1), m\left(V_{L}, D\right)$ is simply a product of a sampling volume $v$ and the total number of samples $N(v, r)$; that is, it is equal to the total volume $V_{L}$ where drops are collected. In this case, the generalized drop concentration $\rho(r)$ coincides with the classical one, which is the mean number of drops per unit volume. For large drops $(r>14 \mu \mathrm{m}, D<1)$ the situation is more complex: not only does the value of $m\left(V_{L}, D\right)$ go down with $D$, but also its unit alters with the drop radius. For these drops, the unit of $m\left(V_{L}, D\right)$ is $\left(\mathrm{cm}^{3}\right)^{D(r)}$. The fractional power indicates that drops with radius $r$ form a clustered set, or fractal (see black dots in Fig. 4). Its Hausdorff-Besicovitch dimension $p$ (Barnsley 1993, p. 198) is $3 D(r)$. Each volume $v$ containing drops is treated as a cluster of size $v^{D(r)}$. The total size of the clustered set in $V_{L}$ is given by the Hausdorff p-dimensional measure (Barnsley 1993, p. 198), which is simply a sum of the cluster sizes over all individual clusters resided in $V_{L}$. In our case, the Hausdorff measure coincides with $m\left(V_{L}, D\right)$. Because only volumes containing drops are counted, $m\left(V_{L}, D\right)$ can be treated as a measure of the "size" of $V_{L}$ after removing "empty spaces" between clusters. The more empty spaces are removed, the lower the value of $m\left(V_{L}, D\right)$ is.

The prefactor $m\left(V_{L}, D\right)$ is a measure of clusters (fractals) but not the number of drops in $V_{L}$. The larger the value of $m\left(V_{L}, D\right)$, the "larger" the cluster. If two sets have different fractal dimensions, then the one with 


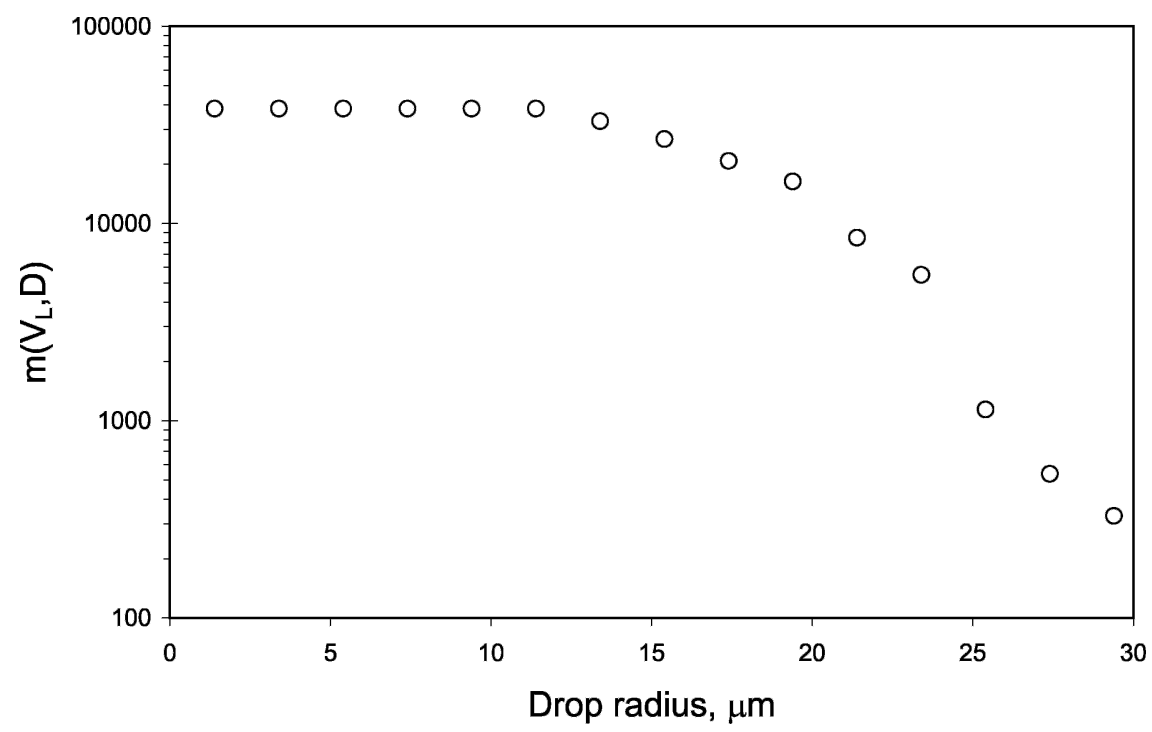

FIG. 5. The dependence of the prefactor $m\left(V_{L}, D\right)$ on the drop radius derived from data described in Fig. 1. For drop radii below $14 \mu \mathrm{m}, D=1$ (see Fig. 3) and $m\left(V_{L}, 1\right)=V_{L}=38272$ $\mathrm{cm}^{3}$. For drop radii above $14 \mu \mathrm{m}, D<1$; the coefficient $m\left(V_{L}, D\right)<m\left(V_{L}, 1\right)$ and its unit is expressed in number of drops per $\mathrm{cm}^{3 D}$.

the higher fractal dimension is the "larger" one (Barnsley 1993, p. 200). The generalized drop concentration, therefore, shows the number of drops per unit cluster. The exponent $D$ determines the degree of clustering: the lower its value, the stronger the clustering and, because of its reduced total size, the less frequent the occurrence of clusters in $V_{L}$ and the higher the generalized drop concentration. Thus with a fixed total number of cloud drops, an increase in clustering (decrease in $D$ ) is accompanied by a corresponding decrease in the occurrence frequency of clusters (increase in $\rho$ ). In other words, for large drops the generalized drop concentration $\rho$ shows the number of drops in a cluster of the size $\left[\mathrm{cm}^{3}\right]^{D(r)}$, and thus one nonempty $\mathrm{cm}^{3}$ contains not $\rho(r)$ but $\rho(r)^{[1 / D(r)]}$ such drops.

Conventional techniques of data processing use samples acquired over the saturation or coarser scales. At these scales, however, the resolution of data does not spatially resolve individual clusters. Once the saturation volume is reached, all sampling volumes will be occupied, resulting in $D=1$. Observed size distributions do not change much with further increases in averaging scale (Liu et al. 2002), and thus the conventional drop concentration can be defined as the ratio of the number of drops in a volume $v \geq v_{\text {sat }}$ to $v$, that is,

$$
c(r)=\frac{\bar{n}(v, r)}{v}=\frac{N_{\mathrm{TOT}}(r)}{m\left(V_{L}, 1\right)}=\frac{N_{\mathrm{TOT}}(r)}{V_{L}}, v \geq v_{\mathrm{sat}} .
$$

The scale independency of $c(r)$ in Eq. (6) is artificially extrapolated from $v \geq v_{\text {sat }}$ down to the smaller scales 0 $<v \leq v_{\mathrm{sat}}$ first and then is used in the classical radiative transfer equation. Information on drop clustering is lost.

Figure 6 shows values of $\rho(\dot{r})^{[1 / D(r)]}$ and the conventional drop concentrations $c(r)$ calculated from Eqs. (5) and (6), respectively, using FIRE data. The following interpretation can be given. For each drop size, the generalized drop concentration accounts for clusters by counting only nonempty volumes. Its value, therefore, depends on the occurrence frequency of clusters parameterized in terms of the exponent $D$. The conventional concentration ignores the clustering by assuming the presence of drops in every volume (i.e., $D=1$ ), which leads to a smaller number of drops in the volume. This is consistent with Eq. (2), which states that for a fixed total number of drops with radius $r$ in a given volume $V_{L}$, a decrease in the occurrence frequency, $N(v, r)$, at a given scale $v$ is accompanied by an increase in the mean number of drops in the nonempty volume $v$.

Results from this section can be summarized as follows: 1) for sufficiently small volumes, the mean number of drops in a given volume is proportional to the volume raised to a drop size-dependent nonunit power; 2) the drop size-dependent coefficient of proportionality, or a generalized drop concentration, shows the mean number of drops per cluster while the power characterizes the occurrence frequency of clusters themselves; 3 ) resolution of data collected at the saturation or coarser scales do not spatially resolve individual clusters and the information on drop clustering is lost. 


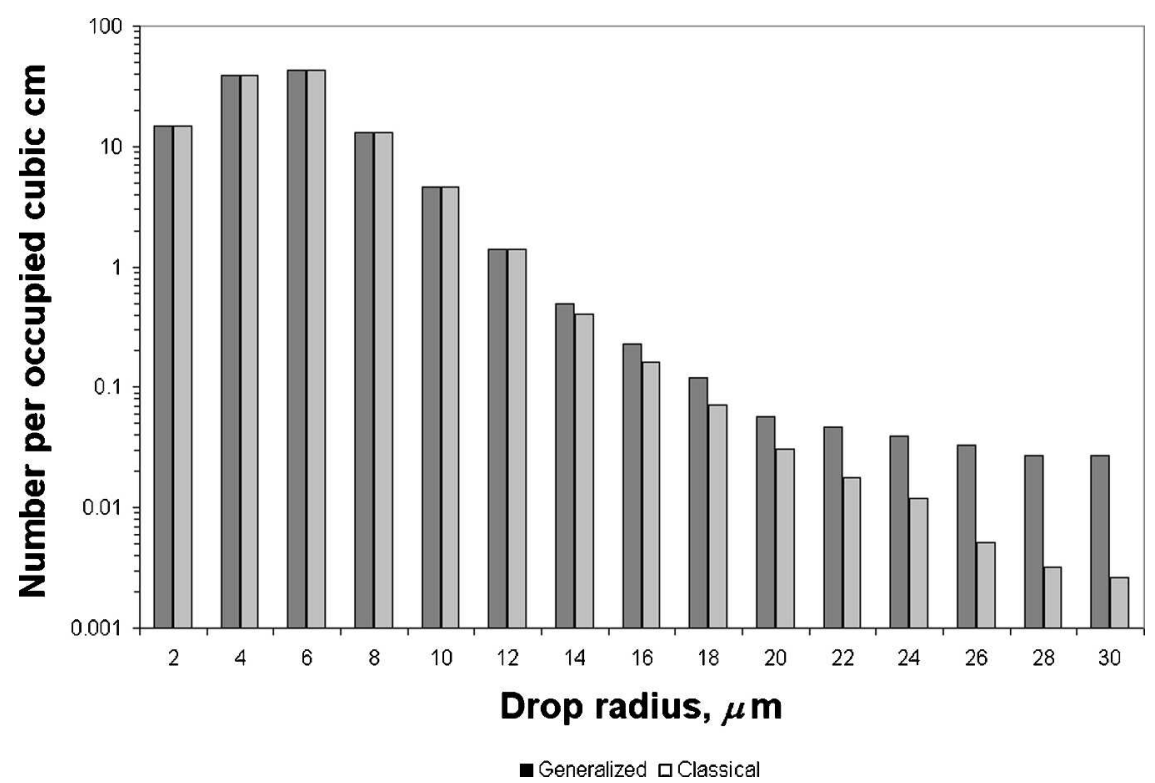

FIG. 6. Mean number of drops in $\mathrm{cm}^{3}$ as a function of drop radius derived from data collected above (legend "Classical") and below (legend "Generalized") the saturation scales. The generalized drop concentration accounts for clusters by counting only volumes filled with drops. Its value, therefore, depends on the occurrence frequency of clusters parameterized in terms of the drop size-dependent exponent $D$ (see Fig. 3). The conventional concentration ignores the clustering by assuming the presence of drops in every $\mathrm{cm}^{3}$ (i.e., $D=1$ ). With a fixed total number of drops in a cloud, a decrease in frequency of clusters is accompanied by a corresponding increase in number of drops per cluster unit.

\section{Impact of drop variability on estimation of cloud optical dimensions}

Our data analysis indicates that, for sufficiently small volumes, the mean number of large drops varies proportionally to the volume raised to a drop sizedependent nonunit power. This finding suggests drop clustering (Marshak et al. 2005). The generalized drop concentration and the nonunit power are two drop sizedependent parameters that characterize the small-scale feature of drop variability. The first one is the mean number of drops per cluster while the second one characterizes the frequency of cluster occurrence. With a fixed total number of cloud drops, a decrease in the frequency of clusters is necessarily accompanied by a corresponding increase in the number of drops per cluster. The conventional drop density concentration is a special case of the generalized drop concentration when the power is set to unity and thus is insensitive to this process. The aim of this section is to estimate the effect of ignoring small-scale drop variability on the estimation of cloud optical properties. This will be accomplished by examining variation in optical distance $\tau(x)$, $0 \leq x \leq L$ along the flight legs. Therefore, we first derive the extinction coefficient, $\sigma(r, D)$, which depends on the drop size $r$ and the exponent $D(r)$; then we derive an equation describing the attenuation of the radiance along the flight leg and examine its solution.

Consider a sample volume $v \in\left[v_{\text {min }}, v_{\text {sat }}\right)$ with a sample area $d S$ and the accumulation distance $d x$, that is, $v=d S d x$. We denote the total interaction cross section $Q_{\mathrm{e}}(r) \pi r^{2}$ by $s_{0}$. Here $Q_{\mathrm{e}}(r)$ is the efficiency factor. The ratio $d S / s_{0}$ is the number $n$ of fine horizontal "tubes" of the size $d x s_{0}$ in the volume $v$. A photon will necessarily be intercepted within such a tube, if the tube contains a drop. The probability of interception, therefore, is determined by the number of nonempty horizontal tubes. The classical approach $(D=1)$ assumes that the number of nonempty tubes (or drops) in the volume $v$ is proportional to $v$ with the drop concentration $\rho$ as a coefficient of proportionality, that is, $\rho v=$ $\rho d x d S=\rho d x s_{0} n=d \tau n$ where $d \tau=s_{0} \rho d x$ is the optical distance. From here the extinction coefficient $\sigma(r, 1)$, in $\mathrm{cm}^{-1}$, can be specified as

$$
\sigma(r, 1)=Q_{e}(r) \pi r^{2} \rho(r) .
$$

For $0<D(r)<1$, the number of drops in the volume $v$ is proportional to the volume raised to $D(r)$, that is, $\rho(d x d S)^{D}=\rho\left(d x s_{0}\right)^{D} n^{D}$. It coincides with the number of intercepted photons, which is proportional to $n$ raised to a drop size-dependent nonunit power. Varia- 
tion in the number of intercepted photons follows a power law. The coefficient $d \tau=\rho\left(s_{0} d x\right)^{D}$ that accounts for intercepted photons is the optical distance. The extinction coefficient $\sigma(r, D)\left[\right.$ in $\left.\mathrm{cm}^{-D(r)}\right]$ thus takes the form

$$
\sigma(r, D)=\left[Q_{e}(r) \pi r^{2}\right]^{D(r)} \rho(r) .
$$

If $0<D(r)<1$, a distribution function $\sigma(r$, $D)[d x]^{D(r)}$ is called singular (Kolmogorov 1950; Richtmyer 1978, p. 260). As has been shown in the companion paper (Marshak et al. 2005), only singular distributions are able to produce the observed power laws shown in Fig. 2. The extinction of radiation by a single drop $[D(r)=0]$ is described by a Heaviside function $H$ and the drop extinction cross section; it is detailed in Knyazikhin et al. (2002).

The removal $d I(x)$ of energy from $I(x)$ along the flight leg due to photon-droplet interaction is

$$
d I(x)=-I(x) \sum_{r} \sigma(r, D) \gamma(r, x)[d x]^{D(r)} .
$$

Here $I(x), 0 \leq x \leq L$, is the radiance at point $x$ along the flight leg; $\gamma(r, x)$ is the indicator function of drops, which takes on the value 1 if there are drops with radius $r$ in the volume $v$ at $x$, and 0 otherwise. The extinction coefficients are given by Eqs. (7) and (8); the exponents $D(r)$ used to calculate $\sigma(r, D)$ are shown in Fig. 3a. For simplicity, we excluded the case $D=0$ from our analysis since it was detailed in Knyazikhin et al. (2002). Note that since drops with $r<14 \mu \mathrm{m}[D(r)=1]$ are present in each sample volume $v$ (Fig. 1), their indicator function $\gamma(r, x)=1$ for all $x$ and $r$.

With a fixed total number of cloud drops, a decrease in the power $D$ is accompanied by (i) an increase in the extinction coefficient [through the increase in $\rho(r, D)^{[1 / D(r)]}$; see, e.g., Fig. 6] and (ii) a decrease in the occurrence frequency of clusters ("more zero values" the indicator functions take on; see, e.g., Figs. 1 and 4). The first process enhances the contribution of drops while the second one suppresses it. Equation (9) captures the competition between these two nonlinearly related processes. The conventional drop concentration corresponds to a special case when $D$ is set to 1 for all drop sizes. In this case, Eq. (9) rearranges to the traditional Beer's law. The use of the conventional drop concentration, therefore, makes the radiative transfer equation insensitive to small-scale drop variability.

The solution of Eq. (9) is $I(0) \exp [-\tau(x)]$ where the optical distance $\tau(x)$ is given by (see item 4 in appendix B)

$$
\tau(x)=\sum_{r} \sigma(r, D) q(D) F(x, D) L^{D(r)} .
$$

Here a dimensionless function $q(D)$ is the prefactor
$m\left(V_{L}, D\right)$ (Fig. 5) normalized by $V_{L}^{D}$, and $F(x, D), 0 \leq$ $x \leq L$, is a distribution function for drops with the exponent $D$ (Fig. 7). For small drops $(D=1), F(x, 1)=$ $x / L$; its probability density function is $1 / L$ (appendix B). For large drops $(0<D<1)$, the distribution function $F(x, D)$ is a continuous, nondecreasing function; its derivative-the probability density distribution function-is zero almost everywhere. Such functions are known as singular distributions (Kolmogorov 1950; Richtmyer 1978, p. 260). The Cantor curve (or "devil staircase") is a classical example of a singular function with $D=\log _{3} 2 \approx 0.63$ (Mandelbrot 1982, p. 99; Richtmyer 1978, 259-260). Figure 7 shows $F(x, D)$ for $D=1$ and $D=0.57$ derived from the bin 11 ( $\sim 22 \mu \mathrm{m}$ drops $)$ of the FSSP data acquired at the ARM Cloud IOP (3 March 2000); a distribution function of one isolated very large $\operatorname{drop}(D=0)$ located at $x=350 \mathrm{~km}$ is added for illustration. If one uses the conventional drop concentration [see Eq. (6)], Eq. (10) rearranges to the classical form

$$
\tau(x)=\sum_{r} \sigma(r, 1) q(1) F(x, 1) L=\sum_{r} \sigma(r, 1) x .
$$

This corresponds to a solution of Eq. (9) for a special case when $D(r)$ and $\gamma(r, x)$ are set to 1 for all $r$ and $x$. Since the conventional drop concentration assumes that large and small drops have identical spatial distributions, Eq. (11) accounts for large drops $(D<1)$ artificially including them in smaller concentrations in every sample volume $v$ by setting $D(r)$ and $\gamma(r, x)$ to 1 . Equation (10) releases the assumption of the drop sizeindependent spatial distributions and describes photon interaction with drops actually present in the sample volume by allowing for variation in $D(r)$ and $\gamma(r, x)$.

To estimate the effect of small-scale drop variability, we compare optical distance calculated with Eqs. (10) and (11) assuming that the drop concentration, both classical and generalized, and $q(D)$ do not depend on the total volume $V_{L}$ in which drops are collected. The distribution function $F(x, D)$ becomes scale invariant in this case (see also item 3 in appendix B), that is,

$$
F(k x, D)=k^{D} F(x, D), k>0 .
$$

The "devil staircase" is a classical example of the distribution satisfying this condition (Mandelbrot 1982, p. 99). Under the above assumption data collected along the 96-km flight leg can be treated as 96 realizations of drop distributions within a 1-km interval. The distribution function is defined on the interval between 0 and 1 km; $F(0, D)=0$ and $F(1, D)=1$. In this case Eq. (10) becomes 


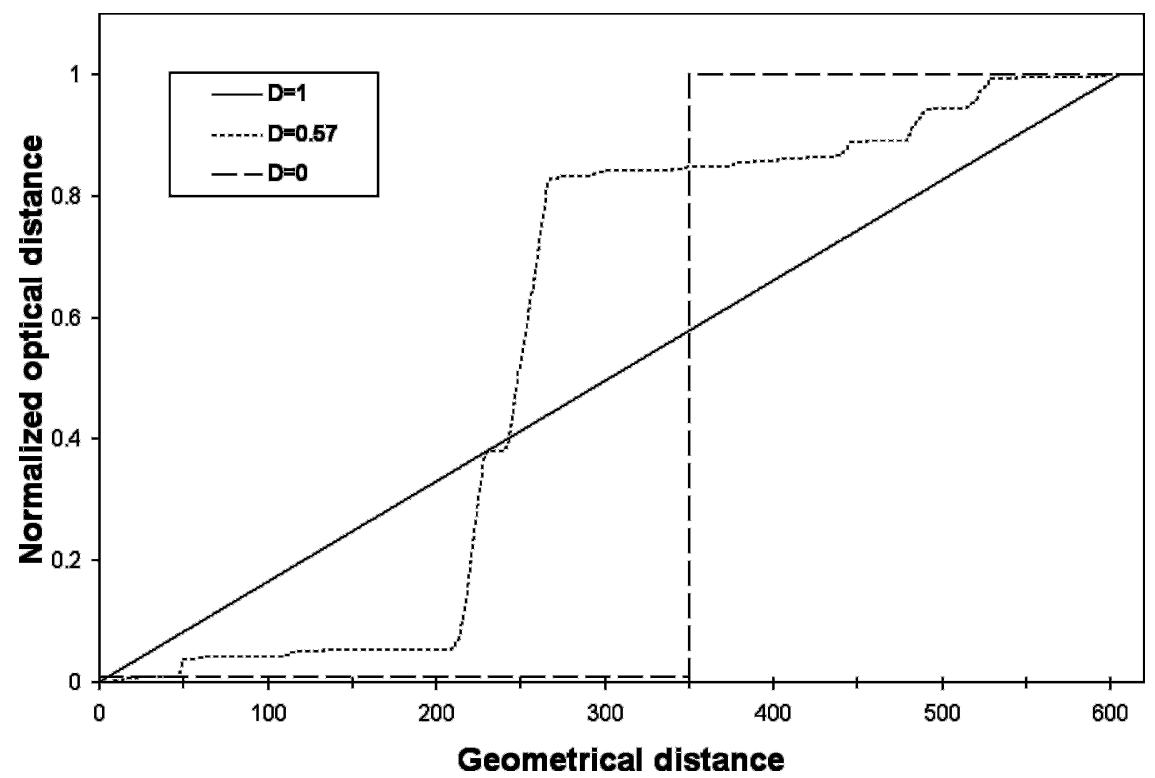

FIG. 7. Distribution function $F(x, D), 0 \leq x \leq 600 \mathrm{~km}$, for drops with the exponent $D=1$ $(r \leq 14 \mu \mathrm{m})$ and $D=0.57(r=24 \mu \mathrm{m})$ derived from data collected during the ARM Cloud IOP on 3 Mar 2000. The distribution function of one isolated drop at $x=350 \mathrm{~km}(D=0)$ is given by a Heaviside function and added for illustration. The function $F(x, D)$ can also be expressed given the solution $I(x)$ of the equation $d I=-I(x) \sigma(r, D)[d x]^{D}$ as follows. Variation in optical distance $\tau(x)$ due drops with the exponent $D$ is $\tau(x)=-\ln [I(x) / I(0)]$. The distribution $F(x, D)$ is then the normalized optical distance $\tau(x) / \tau(600)$.

$$
\tau(x)=\sum_{r} \sigma(r, D) q(D) F(x, D) .
$$

Since $F(x, D)$ is scale invariant, the choice of a $1-\mathrm{km}$ interval as a domain of its definition is not essential to the following analysis, and it has been assumed only for simplicity in notations. Thus it follows from (12) and (13) that the mean optical distance $\tau(L)$ of a longer than $1-\mathrm{km}$ path, $L$, is given by

$$
\tau(L)=\sum_{r} \sigma(r, D) q(D) F(L, D)=\sum_{r} \sigma(r, D) q(D) L^{D(r)}
$$

If the exponent $D$ is set to 1 for all drop sizes, Eq. (14) coincides with Eq. (11) at $x=L$.

To estimate the impact of ignoring a realistic spatial distribution of large drops, we use the ratio of the optical distance resulting from the $14 \mu \mathrm{m}$ and larger drops $(D<1)$ to the total optical distance. Figure 8 demonstrates the ratio as a function of the pathlength derived using Eqs. (11) and (14). Its value is independent of the pathlength and equal to $4 \%$ under assumptions of the conventional approach. The solution of the classical radiative transfer equation continuously depends on the optical depth, and thus it becomes almost insensitive to the presence of large drops. The ratio that accounts for the spatial distribution of large drops exhibits a different behavior. At small geometrical distances, large drops are responsible for about $40 \%$ of the total optical depth. Their impact decreases with the geometrical length. The contribution made by the $14-\mu \mathrm{m}$ and larger drops to the optical distance of a $100-\mathrm{km}$ horizontal line in the cloud is up to $10 \%$. This also suggests that drops that are rare in occurrence have a stronger effect on the cloud radiation regime than the conventional radiative transfer equation can possibly predict.

\section{Conclusions}

One of the weakest links in conventional cloud radiation models is the way a size distribution of cloud particles is mathematically handled. One first degrades measured data from the spatial scale over which they are sampled to a saturation scale beyond which observed size distributions do not change much with further increases in averaging scale, thus also making spatial distributions of drops drop size-independent. Such a distribution is then used in the radiative transfer equation. This approach avoids the scale dependence of the drop concentration. However, it ignores information on small-scale drop variability. 


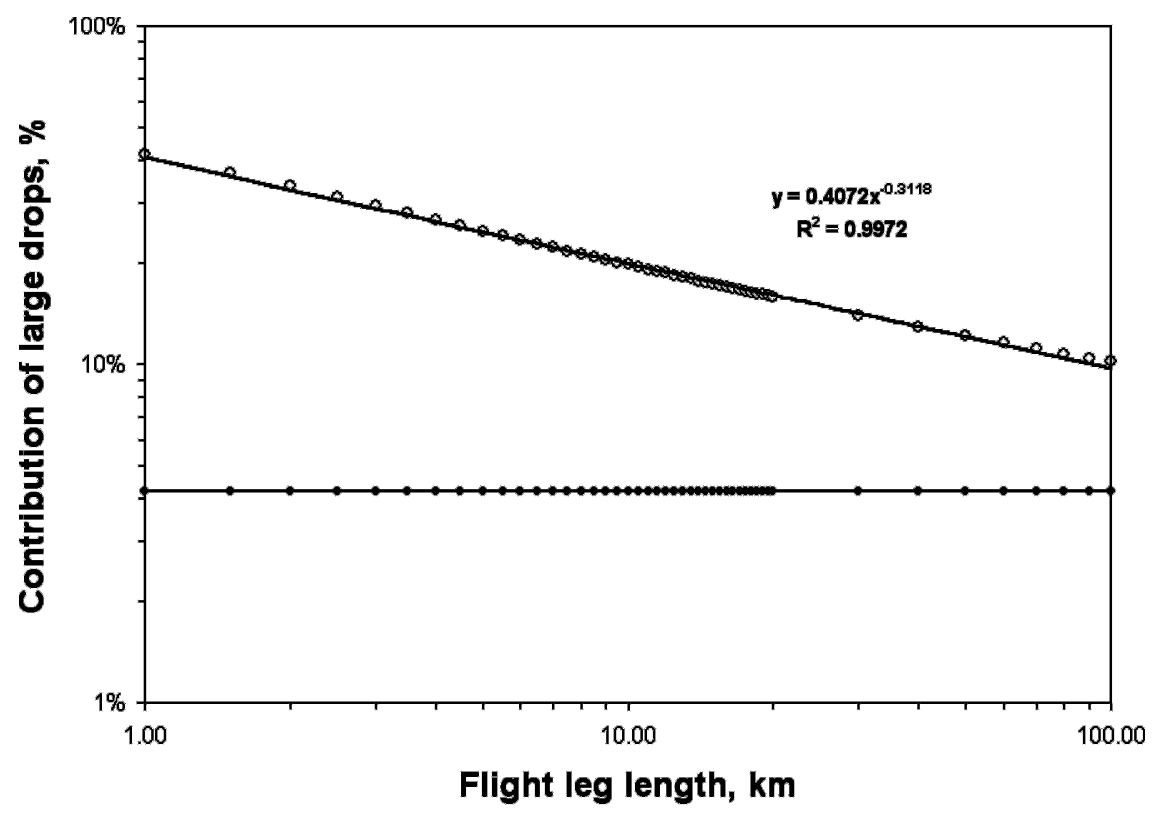

$\rightarrow$ Classical o TRUE —Power (TRUE)

FIG. 8. Contribution of drops with radii between 13.4 and $31.4 \mu \mathrm{m}$ to the optical distance. The ratio of the optical distance for these drops to the total cloud optical distance was calculated using Eqs. (11) and (14) and data collected during the FIRE field program (Fig. 1). The true ratio (open circles) varies with the geometrical distance $L$ as $R(L)=0.41 L^{-0.32}$ (line with open circles). If coarse-resolution data are used, the ratio (line with closed circles) is independent on $L$ and takes on the value $4 \%$.

The analysis of cloud liquid water drop sizes indicates that, for sufficiently small volumes, the mean number of large drops in a given ("nonempty") volume is proportional to the volume raised to a drop size-dependent nonunit power. The coefficient of proportionality, or a generalized drop concentration, is a function of the drop size. The power of the volume, the exponent $D(r)$, is a decreasing function with respect to the drop radius $r$; it varies between 0 and 1 . Models that simulate the observed power law show drop clustering, with the larger drops exhibiting a greater degree of clustering (Marshak et al. 2005). The degree of clustering is determined by the observed exponents $D(r)$ : the smaller its value, the stronger the clustering. For drops of radius $r$, the generalized concentration shows the number of drops per unit cluster and is expressed in $\left(\mathrm{cm}^{3}\right)^{D(r)}$. If $D=1$, cloud drops are not clustered and the generalized drop concentration coincides with the conventional one. With a total number of cloud drops of radius $r$ constant, the deviation of the exponent from unity reduces the frequency of cluster occurrence but increases the drop concentration per cluster. The latter enhances the impact of large drops.

We derived an equation that describes the attenuation of the radiance along a photon path through real- istically distributed cloud drops. It is parameterized in terms of the generalized drop concentration and the exponent. We found that its solution depends on the number of drops per unit cluster rather than per volume. With a total number of (large) cloud drops constant, clustered drops $(D<1)$ have a stronger radiative impact compared to their unclustered counterpart $(D=$ 1 ). If the exponent is equal to 1 , the equation coincides with the traditional radiative transfer equation. If the exponent falls below unity, the use of the traditional equation can result in systematic underestimation of the contribution of large drops to cloud optical path. The following mechanism, missed in the conventional approach, is primarily responsible for this effect. For sufficiently small occupied volumes, the mean number of large drops in a given volume decreases as volume raised to a drop size-dependent nonunit power, while conventional approaches assume a linear decrease with respect to the volume. The latter is a faster process! With a total number of cloud drops constant, the assumption underlying conventional approaches leads to underestimation of the number of rarer large drops per cluster at small scales and, consequently, their impact on radiative transfer. Although the impact decreases with scale, the contribution of large drops to cloud op- 
tical path remains stronger than conventional radiative transfer predicts.

Data on drop size distributions collected at a resolution below the saturation scale is the necessary condition to specify the clustering. A spatial resolution of 80 $\mathrm{m}$ appeared to be sufficient to detect clusters of large drops. Our statistical approach allows us to process much finer resolution data. We believe that the use of very fine resolution data-for example, measured by the Fast FSSP (Pawlowska and Brenguier 1997; Brenguier et al. 1998) — is critical not only to better describe the clustering and its impact on clouds but also to specify physical processes causing this phenomenon.

Acknowledgments. Y. Knyazikhin, A. Marshak, and W. Wiscombe have been supported by the Department of Energy (under Grant DE-A105-90ER61069 to NASA's GSFC) as part of the Atmospheric Radiation Measurement (ARM) Program. The first two authors are also supported by the NASA Radiation Science Program under Grant NNG04GF15G to Boston University. M. Larsen was supported by the Department of Defense's "National Defense Science and Engineering Graduate Fellowship Program," NSF Grant ATM010106271, and the Michigan Space Grant Consortium. He also thanks the Goddard Summer Student Program for its support of this research and his stay at NASA GSFC for the summer of 2003. We also thank the anonymous reviewer for her/his stimulating discussions, which led to a substantial improvement of the paper.

\section{APPENDIX A}

\section{Solution of Eq. (9)}

The aim of this appendix is to derive a solution, $I(x)$, $0 \leq x \leq L$, to Eq. (9). For ease of analysis, we assume only three terms in its right-hand side corresponding to $D\left(r_{1}\right)=1, D\left(r_{2}\right)=D, 0<D<1$, and $D\left(r_{3}\right)=0$. Its solution can be easily generalized to the solution of Eq. (9). Thus, consider the following boundary value problem describing attenuation of the radiance by small $(D$ $=1)$, large $(0<D<1)$, and very large $(D=0)$ drops

$$
\begin{aligned}
d I(x)= & -\sigma_{\mathrm{S}} I(x) d x-\sigma_{L} \gamma_{L}(x) I(x)[d x]^{D} \\
& -\sigma_{\mathrm{VL}} \gamma_{\mathrm{VL}}(x) I(x), \\
I(0)= & i_{0} .
\end{aligned}
$$

Here the extinction coefficients $\sigma_{S}$ (in $\mathrm{cm}^{-1}$ ), $\sigma_{L}$ (in $\mathrm{cm}^{-D}$ ), and the drop extinction cross section $\sigma_{\mathrm{VL}}$ do not depend on $x ; \gamma_{L}\left(\gamma_{\mathrm{VL}}\right)$ is the indicator function of large (very large) drops, which takes on the value 1 if there is a large (very large) drop at $x$, and 0 otherwise. We assume that there is only one "very large" drop at the point $\bar{x}$ in the interval $(0, L)$, that is, $\gamma_{\mathrm{VL}}=1$ if $x=\bar{x}$, and 0 otherwise.

We partition the interval $[0, L]$ into $n$ equal subintervals by subdivisions at $x_{k}=k \delta, k=0$ to $n$, where $\delta=$ $L / n$. We approximate the solution of the Eq. (A1) by a piecewise constant function $I(\delta, x)$; that is, $I(\delta, x)=I_{\delta, k}$ if $x \in\left(x_{k}, x_{k+1}\right)$. Values of $I(\delta, x)$ at $x_{k}=k \delta, k=0, \ldots$, $n$, satisfy the following system of equations:

$$
\begin{aligned}
I_{\delta, k+1}-I_{\delta, k}= & -\sigma_{\mathrm{S}} I_{\delta, k} \delta-\sigma_{L} \gamma_{\mathrm{L}, k} I_{\delta, k} \delta^{D}-\sigma_{\mathrm{VL}} \gamma_{\mathrm{VL}, k} I_{\delta, k}, \\
I_{\delta, 0}= & i_{0} .
\end{aligned}
$$

Here $\gamma_{\mathrm{L}, k}$ and $\gamma_{\mathrm{VL}, k}$ are indicator functions of the interval $\left[x_{k}, x_{k+1}\right)$. In the limit $\delta \rightarrow 0$,

$$
I(x)=\lim _{\delta \rightarrow 0} I(\delta, x) .
$$

Note that Eq. (A3) describes attenuation of radiation along the flight leg where $\delta=l_{\min }=80 \mathrm{~m}, n=1196, L$ $=50 \mathrm{~km}$, and $\left[x_{k}, x_{k+1}\right), k=0, \ldots, n$, represent intervals of data accumulation. Data acquired during the FIRE field experiment allow us to specify all coefficients that appeared in this equation. The length $\delta=$ $l_{\text {min }}=80 \mathrm{~m}$ of the interval over which drops were accumulated is sufficiently small compared to $L$, and thus solutions of Eqs. (A1) and (A3) are not significantly distinct.

It follows from Eqs. (A3) and (A4) that

$$
I_{\delta, k}=i_{0} \prod_{i=1}^{k}\left(1-\sigma_{\mathrm{S}} \delta-\sigma_{L} \gamma_{\mathrm{L}, i-1} \delta^{D}-\sigma_{\mathrm{VL}} \gamma_{\mathrm{VL}, i-1}\right)
$$

The solution $I(\delta, x)$ at $x=L$ can be expressed as

$$
\begin{aligned}
I(\delta, L) & =I_{\delta, n} \\
& =i_{0} \prod_{i=1}^{n}\left(1-\sigma_{S} \delta-\sigma_{L} \gamma_{\mathrm{L}, i-1} \delta^{D}-\sigma_{\mathrm{VL}} \gamma_{\mathrm{VL}, i-1}\right) .
\end{aligned}
$$

Let $N(\delta)$ be the number of subintervals containing large drops $(0<D<1)$. We assume that it follows the power law when $\delta \rightarrow 0$ [cf. with Eq. (3)]:

$$
N(\delta)=f(L, D) \delta^{-D} \text {. }
$$

Here $f(L, D)$ is the prefactor that depends on $D$ and the length $L$ of the interval $[0, L]$. Substituting $\delta=L / n$ into equation for $N(\delta)$ one can obtain

$$
\lim _{n \rightarrow \infty} \frac{N(\delta)}{n}=\lim _{n \rightarrow \infty} \frac{f(L, D) n^{D}}{L^{D} n}=0 .
$$

Note that the product of $n$ factors in the right-hand side 
of Eq. (A6) consists of one factor having the form (1 $\left.\sigma_{S} \delta-\sigma_{L} \gamma_{\mathrm{L}, \mathrm{m}} \delta^{D}-\sigma_{\mathrm{VL}}\right) ; N(\delta)[$ or $N(\delta)-1]$ factors $(1$ $\left.-\sigma_{S} \delta-\sigma_{L} \delta^{D}\right)$, and $n-N(\delta)-1$ [or $n-N(\delta)$ ] factors $\left(1-\sigma_{S} \delta\right)$. Equation (A6), therefore, can be rewritten as

$$
I(\delta, L)=p_{\mathrm{VL}}(\delta, L) p_{L}(\delta, L) p_{S}(\delta, L) i_{0}
$$

where

$$
\begin{gathered}
p_{\mathrm{VL}}(\delta, L)=\left(1-\sigma_{S} \delta-\sigma_{L} \gamma_{\mathrm{L}, \mathrm{m}} \delta^{D}-\sigma_{\mathrm{VL}}\right), \\
p_{L}(\delta, L)=\left(1-\sigma_{S} \delta-\sigma_{L} \delta^{D}\right)^{N(\delta)} \\
p_{\mathrm{S}}(\delta, L)=\left(1-\sigma_{S} \delta\right)^{n-N(\delta)-1}
\end{gathered}
$$

Our next step is to find limits $p_{\mathrm{VL}}(L), p_{L}(L)$, and $p_{\mathrm{S}}(L)$ as $\delta \rightarrow 0$. For very large drops, we have

$$
\begin{aligned}
p_{\mathrm{VL}}(L) & =\lim _{\delta \rightarrow 0} p_{\mathrm{VL}}(\delta, L) \\
& =\lim _{\delta \rightarrow 0}\left(1-\sigma_{S} \delta-\sigma_{L} \delta^{D}-\sigma_{\mathrm{VL}}\right)=1-\sigma_{\mathrm{VL}} .
\end{aligned}
$$

Tending $\delta \rightarrow 0$ in Eq. (A9) and accounting for Eq. (A7) results in the following equation for small drops:

$$
\begin{aligned}
p_{S}(L) & =\lim _{\delta \rightarrow 0} p_{S}(\delta, L)=\lim _{\delta \rightarrow 0}\left(1-\sigma_{S} \delta\right)^{n-N(\delta)-1} \\
& =\lim _{\delta \rightarrow 0}\left(1-\sigma_{S} \delta\right)^{n}=\lim _{\delta \rightarrow 0}\left(1-\sigma_{S} \delta\right)^{L / \delta}=\exp \left(-\sigma_{S} L\right) .
\end{aligned}
$$

Substituting $N(\delta)=f(L, D) \delta^{-D}$ into Eq. (A8), one obtains

$$
\begin{aligned}
\ln p_{L}(\delta, L) & =f(L, D) \delta^{-D} \ln \left(1-\sigma_{S} \delta-\sigma_{L} \delta^{D}\right) \\
& \sim f(L, D) \delta^{-D}\left(-\sigma_{S} \delta-\sigma_{L} \delta^{D}\right) \sim-f(L, D) \sigma_{L} .
\end{aligned}
$$

For large drops, therefore, the coefficient $p_{L}$ takes the form

$$
p_{L}(L)=\lim _{\delta \rightarrow 0} p(\delta, L)=\exp \left(-f(L, D) \sigma_{L}\right) .
$$

It follows from Eqs. (A5), (A10), (A11), and (A12) that the solution, $I(x)$, of the boundary value problem (A1)(A2) at $L$ is

$$
I(L)=\left(1-\sigma_{\mathrm{VL}}\right) \exp \left[-\sigma_{S} L-\sigma_{L} f(L, D)\right] i_{0} .
$$

To find a value of $I(x)$ at an arbitrary chosen point $x$, we define the number $N(\delta, x)$ of subintervals in $[0, x)$ containing large drops $(0<D<1)$. The emergence of large drops in the interval $[0, x)$ can be described by the function $f(x, D)$, defined as

$$
f(x, D)=\lim _{\delta \rightarrow 0} N(\delta, x) \delta^{D} .
$$

In terms of these notations, the solution to the boundary value problem can be expressed as

$$
I(x)=\left[1-\sigma_{\mathrm{VL}} H(x-\bar{x})\right] \exp \left[-\sigma_{S} x-\sigma_{L} f(x, D)\right] i_{0},
$$

where $H(x-\bar{x})$ is the Heaviside function.

\section{APPENDIX B}

\section{Properties of the Solution}

1) Let $\sigma_{L}=0$; that is, the second term in the right-hand side of Eq. (A1) is not present. The solution of Eq. (A1) coincides with one reported in Knyazikhin et al. (2002).

2) The prefactor $f(L, D)$ can be represented as $f(L, D)$ $=q(D) L^{D}$ where $q(D)=\lim _{\delta \rightarrow 0} N(\delta) n^{-D}$. Indeed, it follows from $N(\delta)=f(L, D) \delta^{-D}$ and $n^{D} \delta^{D}=L^{D}$ that

$$
f(L, D)=\lim _{\delta \rightarrow 0} N(\delta) \delta^{D}=\lim _{\delta \rightarrow 0} \frac{N(\delta)}{n^{D}} n^{D} \delta^{D}=q(D) L^{D} .
$$

3) The emergence of small $(D=1)$ and large $(0<D<$ 1) drops in the interval $[0, x)$ can be described by the cumulative distribution function $F(x, D)$, defined as

$$
F(x, D)=\frac{f(x, D)}{f(L, D)}=\frac{f(x, D)}{q(D) L^{D}} .
$$

This is a nondecreasing, continuous function and $F(0, D)=0, F(L, D)=1$. It follows from (A6) that the increment $d F=F(x+\delta, D)-F(x, D)$ is proportional to $\delta^{D}$. It means that the probability density distribution function $d F / \delta$ cannot be defined if $D<$ 1. For $D=1, F(x, 1)=x / L$ if $x<L$, and $F(x, 1)=$ 1 if $x \geq L$.

4) The solution of Eq. (A1) can be expressed in terms of the cumulative distribution function as

$$
I(x)=\left[1-\sigma_{\mathrm{VL}} H(x-\bar{x})\right] \exp [-\tau(x)] i_{0},
$$

where the optical distance $\tau(x)$ is given by

$$
\tau(x)=\sigma_{S} F(x, 1) L+\sigma_{L} F(x, D) q(D) L^{D} .
$$

\section{REFERENCES}

Albrecht, B. A., D. A. Randall, and S. Nicholls, 1988: Observations of marine stratocumulus clouds during FIRE. Bull. Amer. Meteor. Soc., 69, 618-626.

Ash, R., 1965: Information Theory. Dover, 339 pp.

Barnsley, M. F., 1993: Fractals Everywhere. 2d ed. Academic Press, $531 \mathrm{pp}$.

Brenguier, J. L., T. Bourrianne, A. de Araujo Coelho, J. Isbert, R. Peytavi, D. Trevarin, and P. Weschler, 1998: Improvements of droplet size distribution measurements with the Fast-FSSP (Forward Scattering Spectrometer Probe). J. Atmos. Oceanic Technol., 15, 1077-1090.

Dong, X., P. Minnis, G. G. Mace, W. L. Smith Jr., M. Poellot, 
R. T. Marchan, and A. D. Rapp, 2002: Comparison of stratus cloud properties deduced from surface, GOES, and aircraft data during the March 2000 ARM Cloud IOP. J. Atmos. Sci., 59, 3265-3284.

King, M. D., L. F. Radke, and P. V. Hobbs, 1990: Determination of the spectral absorption of solar radiation by marine stratocumulus clouds from airborne measurements within clouds. J. Atmos. Sci., 47, 894-907.

Knyazikhin, Y., A. Marshak, W. J. Wiscombe, J. V. Martonchik, and R. B. Myneni, 2002: A missing solution to the transport equation and its effect on estimation of cloud absorptive properties. J. Atmos. Sci., 59, 3572-3585.

Kolmogorov, A. M., 1950: Foundations of the Theory of Probability. Chelsea, $71 \mathrm{pp}$.

Liu, Y., and J. Hallett, 1998: On size distributions of cloud droplets growing by condensation: A new conceptual model. J. Atmos. Sci., 55, 527-536.

- , P. H. Daum, and J. Hallet, 2002: A generalized systems theory for the effect of varying fluctuations on cloud droplet size distribution. J. Atmos. Sci., 59, 2279-2290.

Mandelbrot, B. B., 1982: The Fractal Geometry of Nature. W. H. Freeman, $460 \mathrm{pp}$.

Marshak, A., Y. Knyazikhin, M. Larsen, and W. J. Wiscombe, 2005: Small-scale drop size variability: Empirical models for drop size-dependent clustering in clouds. J. Atmos. Sci., 62, $551-558$.

Pawlowska, H., and J. L. Brenguier, 1997: Optimal nonlinear estimation of cloud particle measurements. J. Atmos. Oceanic Technol., 14, 88-104.

Richtmyer, R. D., 1978: Principles of Advanced Mathematical Physics. Vol. 1. Springer-Verlag, 422 pp. 